\title{
Lightcurve of asteroid Nereus
}

\author{
Yukihiro Ishibashi ${ }^{1,2}$, Masanao $\mathrm{Abe}^{2}$, and Yasuhiko Takagi ${ }^{3}$ \\ ${ }^{1}$ Graduate School of Science, University of Tokyo, 7-3-1 Hongo, Bunkyo, Tokyo 113-0033, Japan \\ ${ }^{2}$ Institute of Space and Astronautical Science, 3-1-1 Yoshinodai, Sagamihara, Kanagawa 229-8510, Japan \\ ${ }^{3}$ Toho Gakuen Junior College, 3-11 Heiwagaoka, Meito, Nagoya 465-8515, Japan
}

(Received June 2, 1999; Revised June 12, 2000; Accepted June 19, 2000)

\begin{abstract}
An Apollo asteroid (4660) Nereus is one of the most accessible asteroids by spacecraft. Photometric observations of light variation of Nereus were performed in order to support a mission scenario planning and to elucidate the origin of the asteroid. The results revealed that Nereus has a long rotational period, $15.1 \pm 1.2$ hours, and that the amplitude of light variation is fairly large. Its slow rotation is preferable for a landing or touch down scenario of spacecraft. On the other hand, the comparison of lightcurve data between small main-belt asteroids and comet nuclei suggests that Nereus may be an extinct cometary nucleus, rather than a migrated asteroid from the main-belt. The comparison of the observed data with laboratory impact experiments also implies that the asteroid may not be a fragment from a catastrophic impact event in the main-belt. All the other data already obtained, such as by colorimetry, are not inconsistent with the cometary origin, either.
\end{abstract}

\section{Introduction}

An Apollo asteroid (4660) Nereus was discovered in 1982 by E. F. Helin (Helin et al., 1984). Since its inclination $\left(1.4^{\circ}\right)$ and eccentricity $(0.360)$ are small and its perihelion distance $(0.95 \mathrm{AU})$ is near to $1 \mathrm{AU}$, Nereus is recognized as one of the most accessible asteroids by spacecraft (Helin et al., 1984; Davis et al., 1993; Abe et al., 1996). Therefore, it was the backup target of both the NEAR mission of NASA (Farquhar et al., 1995) and the ROSETTA mission of ESA (Lauer, 1992). It was also considered as the original target for the MUSES-C mission of ISAS, the first asteroid sample return mission in the world (Kawaguchi et al., 1996; Fujiwara et al., 2000), and the NEAP mission of SpaceDev Inc., the first private planetary exploration.

To plan a mission scenario of such explorations, understanding of physical properties of the target body such as size, shape, rotational period, and pole (rotational axis) orientation, are vital. For example, the mission scenario of NEAR was planned based on the well-known properties (sidereal period, pole orientation, size, shape, phase coefficient, geometric albedo, and taxonomic type) of the target, (433) Eros (Yeomans, 1995). Images obtained during the NEAR spacecraft's flyby in December 1998 revealed that the data obtained by ground-based observations were in a good agreement (Murchie et al., 1999).

On the other hand, available data on Nereus before the last opposition of 1997 summer were very limited. The observed data on the absolute magnitude and the visible spectrum were still preliminary. Some preliminary photometric data were also compiled by Ephemerides of Minor Planets (EMP) (Batrakov, 1996). The absolute magnitude in V band

Copy right (C) The Society of Geomagnetism and Earth, Planetary and Space Sciences (SGEPSS); The Seismological Society of Japan; The Volcanological Society of Japan; The Geodetic Society of Japan; The Japanese Society for Planetary Sciences.
$\left(H_{\mathrm{V}}(1,1,0)\right)$ is reported as +18.3 . If the albedo is assumed to be 0.05 , the diameter can be estimated as $1.3 \mathrm{~km}$. Tholen and Senay (1993) performed a visible colorimetry observation in 6 colors during the opposition in 1993. The spectrum obtained is featureless and flat (Tholen and Whiteley, 1998). Other physical properties necessary to plan the spacecraft mission were totally unknown.

In the case of Eros, numerous radar data were available for planning of the NEAR mission (Ostro et al., 1990). Radar observations for small $(<1 \mathrm{~km})$ asteroids can be performed only for distances to asteroids less than $0.05 \mathrm{AU}$, and the number of facilities that can be used for radar observations is limited. On the contrary, photometric observations of light variation can easily be performed for many asteroids including Nereus. Physical properties, such as shape, rotational period, and pole orientation, can be deduced by evaluating the light variation. Therefore, ground-based photometric observations are the first step in order to start planning for a mission to Nereus.

In the astronomical time scale, near-Earth objects (NEOs) including Nereus are rapidly removed from current orbits by collisions with planets and other asteroids, or by dynamical ejection from the Solar System. Since lifetimes of these bodies in the inner Solar System (10-100 Myr) are shorter than the age of the Solar System (Bottke et al., 1994), continuous sources are necessary. Suggested sources are main-belt asteroids and extinct or dormant comet nuclei (Tedesco and Gradie, 1987; Wetherill, 1988; Binzel et al., 1992a; Lupishko and Martino, 1998). Michel et al. (1996) and Yoshikawa et al. (2000) calculated the orbital evolution of Nereus using "clone" asteroids. Their results were not conclusive on the origin of Nereus, since its orbit is chaotic due to close encounters with terrestrial planets, whereas the result for Eros suggested a "slow-track" evolution from the main belt (Michel 
Table 1. Observational conditions of (4660) Nereus.

\begin{tabular}{|c|c|c|c|c|c|c|c|}
\hline \multirow{2}{*}{$\begin{array}{l}\text { Date } \\
\text { (UT0) }\end{array}$} & \multicolumn{4}{|c|}{ R. A. (J2000) Decl. } & \multirow{2}{*}{$\begin{array}{c}r \\
(\mathrm{AU})\end{array}$} & \multirow{2}{*}{$\begin{array}{c}\Delta \\
(\mathrm{AU})\end{array}$} & \multirow{2}{*}{$\begin{array}{c}\alpha \\
\text { (degree) }\end{array}$} \\
\hline & $\mathrm{h}$ & $\mathrm{m}$ & $\circ$ & , & & & \\
\hline 3 Aug. 1997 & 19 & 42.67 & -21 & 57.9 & 2.011 & 1.018 & 8.5 \\
\hline 4 Aug. 1997 & 19 & 41.08 & -21 & 00.8 & 2.010 & 1.021 & 9.2 \\
\hline
\end{tabular}

$r$ : heliocentric distance, $\Delta:$ geocentric distance, $\alpha:$ phase angle of Sun-Nereus-Earth

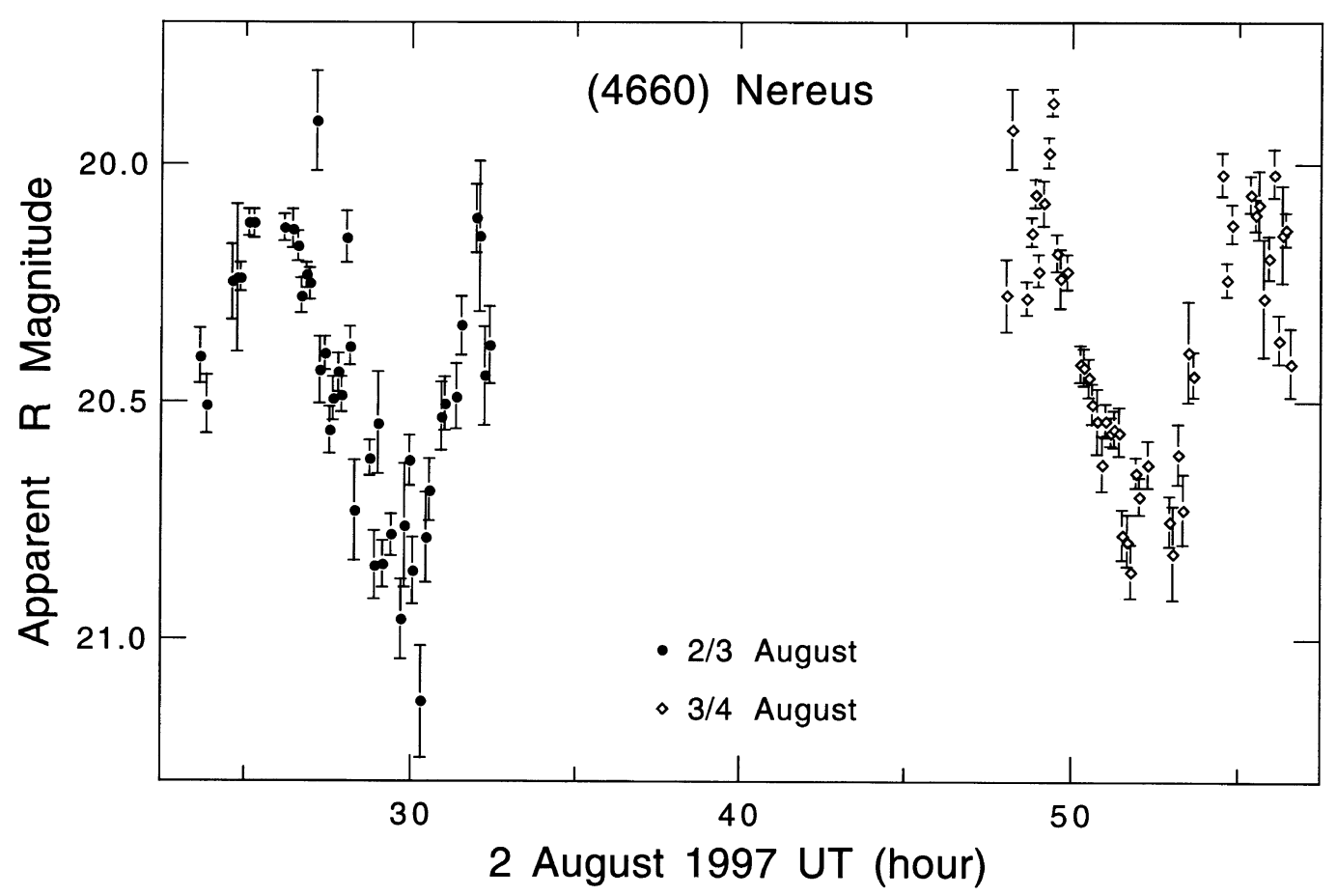

Fig. 1. Observed apparent magnitude in R band of (4660) Nereus.

et al., 1996).

Some meteors with orbits similar to Nereus were also observed (Olsson-Steel, 1988; Babadzhanov, 1994; Ohtsuka et al., 1995). These meteors are supportive evidence for its cometary origin. However, they are not still conclusive, since meteors associated with typical S and Q-type asteroids, such as (433) Eros and (1864) Apollo, are also reported (Babadzhanov, 1994). From other data already obtained on Nereus, any implication on its origin has not been inferred.

Binzel et al. (1992a) compiled data on the rotational frequency and light variation amplitude of small $(<5 \mathrm{~km})$ mainbelt asteroids (SMBAs) and comet nuclei. They showed that cometary nuclei have longer rotational period and larger amplitude of light variation than SMBAs have, and evaluated the fraction of comet origin in NEOs as 0 to $40 \%$. Recently, Lupishko and Martino (1998) compiled the latest data of absolute magnitude, albedo, taxonomic type, rotational period, light variation amplitude, and colors (U-B and B-V) on near-Earth asteroids. They concluded that the main source of near-Earth asteroids is the main belt asteroids. Although the used datasets were not complete and their conclusions were not definitive, their results imply that the lightcurve data on NEOs is a key to discuss their origins.
With the above background in mind, we performed lightcurve observations of Nereus during the last opposition in August 1997, in order to obtain its rotational period and amplitude of light variation.

\section{Observations}

Photometric observations were performed in the nights of August 2/3 and 3/4, 1997, using the ESO/MPI 2.2-m telescope at La Silla. The ESO \#40 CCD and an R Bessel broadband filter were used. The exposure time was 300 seconds. Data coverage was about 8.5 hours per night. The sky condition was good over the whole observation period. Observational conditions are listed in Table 1.

After carefully subtracting bias and flat-fielding, the aperture photometry technique using IRAF software (Tody, 1986) was applied. Effects from field stars around Nereus were also carefully removed for the data that were affected by them, but still some other data could not be used since these effects were too large to be corrected. Then the relative photometry was made between Nereus and comparison stars in the same frame, in addition to standard stars (Landolt, 1992) in another frame. Eleven comparison stars appeared near Nereus were measured in each frame to make relative photometry 
with Nereus, and to monitor the background sky variation. Apparent magnitude of selected comparison stars are from 15 , which is about 5 magnitude brighter than Nereus, to 21 , which is about 1 magnitude fainter. Their photometric errors are less than 0.005 magnitude for brighter ones in all frames, and up to about 0.07 magnitude for the faintest one in most frames. It is confirmed that brightness variation among comparison stars are less than 0.01 magnitude. Four standard stars (Landolt, 1992) were also used to derive the atmospheric coefficient on each day. Finally, the apparent magnitude was derived by correcting the airmass. The obtained lightcurve is shown in Fig. 1.

The absolute magnitudes $\left(H_{\mathrm{R}}(1,1,0)\right)$ were calculated using the method by Bowell et al. (1989) and the slope parameter, $G$, was assumed to be 0.15 .

\section{Analyses}

The period of Nereus' light variation was analyzed by two different methods. One is the least-squares frequency analysis method (Lomb, 1976; Press et al., 1992), and another is an independent analysis using a phase dispersion minimization method (Lafler and Kinman, 1964). The first is an application of the Fourier transfer method for unevenly spaced data. The least-squares spectrum obtained by this method is shown in Fig. 2. In total 82 dataset were used for this analysis.

In Fig. 2, three large peaks are indicated at 5.80, 7.54, and 10.90 hours. The wide width of each peak may be due to the much shorter observation time than the full period of the lightcurve. The approximate ratio among these three large peaks at 5.80, 7.54, and 10.90 hours in Fig. 2 is $\frac{1}{4}: \frac{1}{3}: \frac{1}{2}$, in that order. The 7.54-hour period allows us to draw the lightcurve with two minima within a gap between two sets of observed data obtained in the nights of the 2nd and the 3rd of August. Similarly, both 5.80- and 10.90-hour periods have three minima and one minimum, respectively, in the same gap. The false-alarm probability of the null hypothesis for the least-squares method is approximately expressed by

$$
P(>z) \propto M e^{-z},
$$

where $M$ is a constant and $z$ is the power (Press et al., 1992). Since the estimate of $M$ is not straightforward, relative values of $P$ given in Fig. 2 as dashed lines were calculated with Eq. (1). Three lines in Fig. 2 indicate that the significance level of the central peak is three orders of magnitude higher than those of other peaks. This means that only the central peak, $7.54 \pm 0.6$ hours, is statistically significant period.

The second method applied to the data of Nereus was developed by Lafler and Kinman (1964). This method takes into account of smoother and consequent light variation. The parameter to express smoothness in this method is the sum of squares of magnitude difference of observation between two adjacent phases, when the data are fold up with a specific period. The parameter is expressed as

$$
\theta=\frac{\sum_{i}\left(H_{i}-H_{i+1}\right)^{2}}{\sum_{i}\left(H_{i}-\bar{H}\right)^{2}},
$$

where $H_{i}$ is the magnitude and $\bar{H}$ is their average. The period which makes the parameter $\theta$ the minimum is considered to

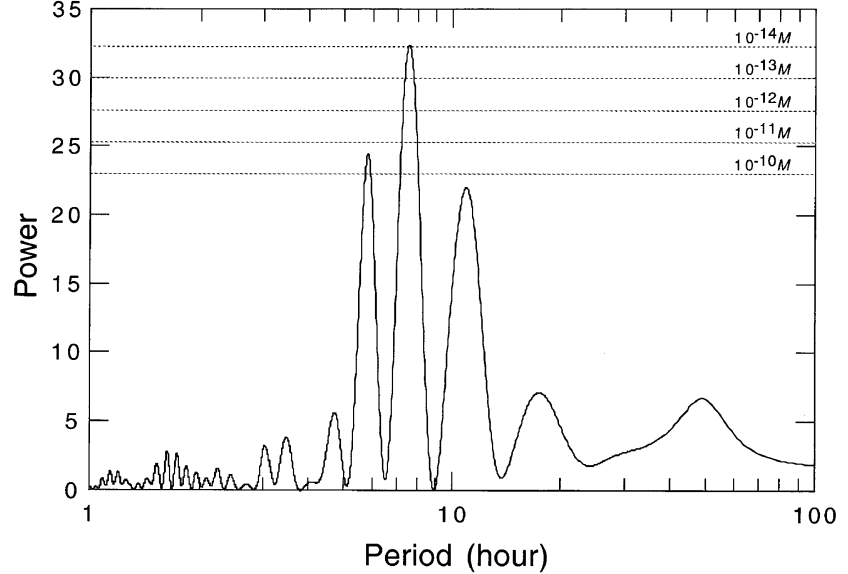

Fig. 2. The least-squares spectrum of $H_{\mathrm{R}}(1,1,0)$ lightcurve of (4660) Nereus (modified from Ishibashi et al. (2000)). Dashed horizontal lines indicate significance levels calculated by Eq. (1), where $M$ is a constant.

be what is desired. The period obtained by the application of this criterion to our data is 7.4 hours. This value is well consistent with that by the least-squares method.

Since lightcurves of asteroids generally have two maxima and two minima in one rotational period, the rotational period of Nereus is estimated to be $15.1 \pm 1.2$ hours, twice the lightcurve period estimated above. This result reconfirmed the result of Tholen and Whiteley (1998), 15.1 hour, whose observation was performed during the same opposition, but in $\mathrm{V}$ band, within a error range of our results by the leastsquares method. Figure 3 shows the lightcurve assuming that the period is 15.1 hours.

Lightcurves of asteroids can be expressed as

$$
I_{r}^{2}=B \cos ^{2}(\Psi)+C
$$

where $I_{r}=10^{-0.4 H_{\mathrm{R}}}$, and $\Psi$ is the phase from the maximum point (Pospieszalska-Surdej and Surdej, 1985). Leastsquares fitting of Eq. (3) to the present data derived that the constants $B$ and $C$ are $(2.90 \pm 0.16) \times 10^{-15}$ and $(1.22 \pm$ $0.10) \times 10^{-15}$, respectively. This implies that the mean magnitude is $18.22 \pm 0.06$ and the amplitude of the light variation is $0.66 \pm 0.04$ magnitude. The curve fitted by Eq. (3) is also shown in Fig. 3 as a dashed line.

The amplitude of light variation is known to increase with the phase angle. The relationship between the amplitude and the phase angle was investigated by many works (e.g., Zappalà et al., 1990; Lumme and Bowell, 1981 a and 1981b). As Binzel et al. (1992a) used the relationship yielded by Zappalà et al. (1990), we also adopted this, which is expressed as

$$
A(0)=\frac{A(\alpha)}{(1+m \alpha)}
$$

where $A(0)$ is the amplitude of light variation at phase angle $(\alpha)$ of $0^{\circ}$. The constant $m$ is found to be $0.030,0.015$, and 0.013 degree $^{-1}$ for S, C, and M-type asteroids, respectively. We have chosen the C-type asteroid value for this study because this is consistent with Nereus' featureless and flat spectrum obtained by Tholen and Senay (1993). The average value of $\alpha$ was assumed to be 8.95 degree. Then the 


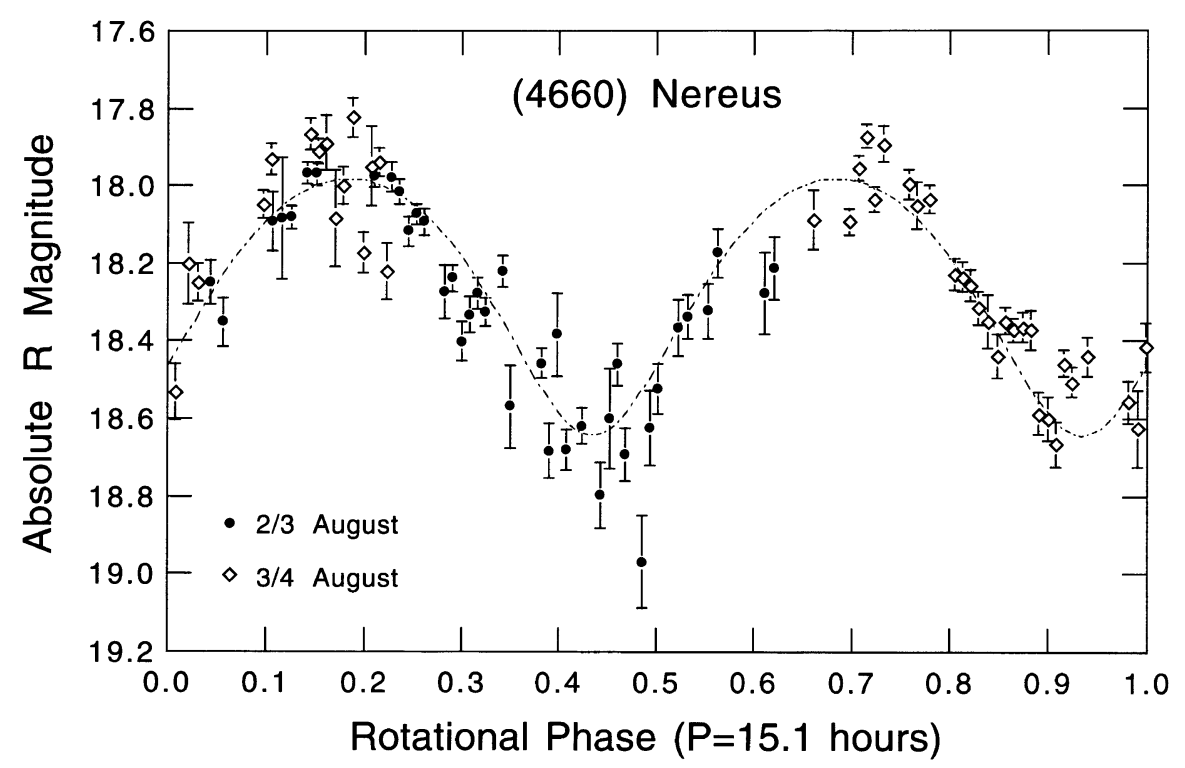

Fig. 3. Obtained lightcurve of (4660) Nereus. The abscissa is phase of 15.1 hours, and the ordinate is the derived absolute magnitude, $H_{\mathrm{R}}$. The dashed curve shows the light variation expressed by Eq. (3).

amplitude of light variation at $\alpha=0^{\circ}$ can be estimated as $0.58 \pm 0.03$ magnitude.

\section{Discussion}

\subsection{Nereus as a spacecraft mission target}

Since our observations were performed only in the R band, the direct comparison with the absolute magnitudes in the $\mathrm{V}$ band compiled by EMP (Batrakov, 1996) is impossible. The featureless and flat spectrum obtained by Tholen and Senay (1993) implies, however, that it is reasonable to assume that the magnitude difference between $\mathrm{V}$ and $\mathrm{R}$ bands of Nereus, $H_{\mathrm{V}}-H_{\mathrm{R}}$, is similar to that of the Sun. If the $H_{\mathrm{V}}-H_{\mathrm{R}}$ is 0.36 , the absolute magnitude of Nereus in the $\mathrm{V}$ band is $18.58 \pm 0.06$. This value is slightly fainter than the value given in EMP. Although the precise comparison cannot be possible, because the original data used by EMP are unknown, this discrepancy may be due to the large amplitude of light variation. The absolute magnitude in EMP is based on data when the light variation was near to its maximum. If the same value of albedo can be assumed, the diameter of Nereus estimated from the present value is about $10 \%$ smaller than the previous albedo value. This correction of the target body size affects to the mission planning in terms of such as total amount of the required fuel.

The long rotational period and large amplitude of light variation obtained by the present observation also have effects on a NEO mission like MUSES-C. The observed rotational period, 15.1 hours, is very preferable both for the landing or touch down scenario of the spacecraft, and for some remote sensing observation, such as X-ray spectrometry. MUSES-C spacecraft is planned to approach toward the asteroid's gravitational center from the Solar direction. At some altitudes from the asteroid surface, say 50 to $100 \mathrm{~m}$, some lateral maneuver proportional to the rotational velocity of the target object will be performed such that the motion of spacecraft is synchronized with the rotation of asteroid. Then the spacecraft will be able to descend to a pre-determined position on the surface slowly. If its rotational period is not enough long, the rotation of the asteroid and the spacecraft synchronizing may cause the off-set of solar cell panel on the spacecraft from the Sun direction and thus decrease of power generation during the final approaching phase. The spacecraft may also lose the landing or touch down point on the surface of the asteroid.

On the other hand, the elongated or irregular shape of Nereus, estimated from the observed lightcurve, is not preferable for the spacecraft landing or touch down. If the albedo on the asteroid surface is uniform, the large amplitude of light variation means the large irregularity of its shape. If the asteroid is elongated perpendicular to the rotational axis, its rotational velocity varies from position to position. The direction of gravity is also deviated from the surface normal vector. The spacecraft navigation can be difficult in such cases.

To plan the mission scenario more reliable, information on its pole orientation is also essential. The large amplitude of light variation obtained suggests a large aspect angle, the angle between Nereus' rotational axis and the line of sight during our observation. This result gives a constraint on the pole orientation for preferable mission target. Therefore, more observations from different aspect angles are desired to determine precise pole orientation.

\subsection{Implication for cometary origin of Nereus}

Binzel et al. (1992a) discussed on the origin of NEOs using compiled data on the rotational frequency and light variation amplitude of small $(<5 \mathrm{~km})$ main-belt asteroids (SMBAs) and comet nuclei, from which those parameters of Nereus can be directly compared. They applied the phase and aspect angle effect corrections to the original data set on the amplitude of light variation. The correction for the phase angle effect was performed by using the relationship derived by Zappalà et al. (1990). The correction for the aspect angle effect was performed using the procedure developed by Binzel and Sauter (1992). The procedure estimates the lightcurve 
Table 2. Nereus' rotational frequency and amplitude of light variation.

\begin{tabular}{ccc}
\hline & $\begin{array}{c}\text { Rotational frequency } \\
\text { (rev/day) }\end{array}$ & $\begin{array}{c}\text { Amplitude of light variation } \\
(\mathrm{mag})\end{array}$ \\
\hline $\begin{array}{c}\text { Small main-belt asteroids } \\
\text { (Binzel } \text { et al., 1992a) }\end{array}$ & $4.30 \pm 0.46$ & $0.269 \pm 0.041$ \\
$\begin{array}{c}\text { Comet nuclei } \\
\text { (Binzel } \text { et al., 1992a) }\end{array}$ & $1.64 \pm 0.39$ & $0.598 \pm 0.081$ \\
Nereus (This work) $^{2}$ & $1.59 \pm 0.13$ & $0.58 \pm 0.03$ \\
\hline
\end{tabular}

${ }^{1}$ Uncertainties are the standard deviation of the mean.

${ }^{2}$ Uncertainties are the observational error.
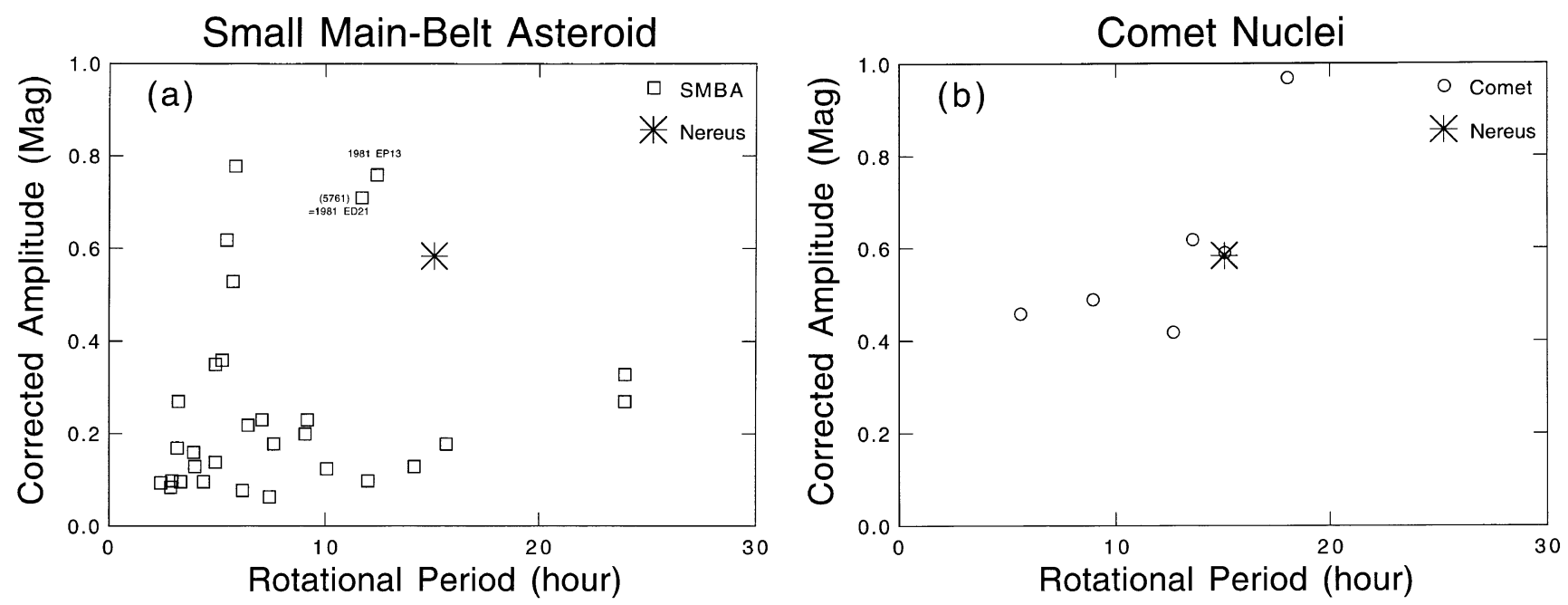

Fig. 4. Rotational period and corrected amplitude of light variation calculated by the procedure of Binzel and Sauter (1992) for small main-belt asteroids (Fig. 4(a)) and for comet nuclei (Fig. 4(b)).

amplitudes as if they were observed at an aspect angle of $60^{\circ}$. Binzel et al. (1992a) performed this procedure and calculated the average values of rotational frequency and corrected amplitude for NEOs, SMBAs, and comet nuclei. The average values for SMBAs and comet nuclei calculated by Binzel $e t$ al. (1992a) are listed in Table 2. We also applied these two corrections to our data of Nereus, which are also listed in the table. Both values of Nereus agree with the averages of comet nuclei within the standard deviation of the mean. On the contrary, the rotational frequency of Nereus is significantly smaller than the average value of SMBAs and the corrected amplitude is significantly larger than the average value of SMBAs. These results suggest that the origin of Nereus may be a comet nucleus, rather than a main-belt asteroid.

In Fig. 4, this implication is more clearly shown by the corrected amplitude-rotational period relation for SMBAs (Fig. 4(a)) and for comet nuclei (Fig. 4(b)). Data are taken from Binzel et al. (1992b) on SMBAs and from Luu and Jewitt (1992) and other references cited in Jewitt (1991) on comet nuclei. The phase angle effects for comet nuclei were calculated using the constant $m$ for C-type asteroids in Eq. (4). On the other hand, all but one of SMBAs were assumed as S-type asteroid. Since the observed value of $H_{\mathrm{V}}-H_{\mathrm{R}}$ in the Johnson system for 1981ET20 was 0.56 (Binzel et al., 1992b), it was assumed as C-type.

Figure 4(a) shows that the property of lightcurve of Nereus is different from those of SMBAs. Most of main-belt asteroids shown in Fig. 4(a) do not have longer rotational periods nor larger amplitudes than Nereus. On the contrary, Fig. 4(b) shows that the rotational period and amplitude of light variation of Nereus are similar to those of comet nuclei. This result also supports that the origin of Nereus may be a comet nucleus.

\subsection{Comparison in shape with impact fragments}

The limit of axis ratio, $a: b: c(a>b>c)$, of a triaxial ellipsoid model of an asteroid can be estimated from the observed amplitude of light variation. The dynamically stable rotational axis of a triaxial ellipsoid is the shortest axis, $c$. When it is seen from the direction perpendicular to the rotational axis, $c$, the axial ratio, $\left(\frac{a}{b}\right)$, is expressed by

$$
\log \left(\frac{a}{b}\right)=0.4 A(0) \text {. }
$$

The $\left(\frac{a}{b}\right)$ from the obtained value of $A(0)=0.58 \pm 0.03$ is then $1.71 \pm 0.05$. Since observations are generally made from an oblique direction, the observed amplitude of light variation should be smaller than the value calculated from Eq. (5). 
Therefore the true ratio $\left(\frac{a}{b}\right)$ of Nereus should be larger than the observed value, 1.71, indicating that the shape of Nereus is fairly elongated perpendicular to the rotational axis.

The comparison of the estimated $\left(\frac{a}{b}\right)$ ratio with results from impact experiments also gives some implications on the origin of Nereus. The triaxial ratios of impact fragments produced by catastrophic disruption were investigated by laboratory impact experiments performed by Fujiwara et al., (1978) and Capaccioni et al., (1984). Both found that the average ratio of $a: b: c$ is approximated by the simple ratio of $2: \sqrt{2}: 1$. The frequency distribution of $\left(\frac{a}{b}\right)$ ratio also indicated that the fraction of fragments whose $\left(\frac{a}{b}\right)$ ratio is larger than 1.71 is about 15\% (Fujiwara et al., 1978). This result implies that Nereus may be not a single fragment from a catastrophic impact in the main-belt. This is also an additional support for the cometary origin of Nereus.

\subsection{Rotational condition}

Burns and Safronov (1973) pointed out that small asteroids with slow rotations can have "damping" time scales of rotational wobbling longer than the age of the Solar System. So far non-principal axis rotations have been identified among three near-Earth asteroids, (3288) Seleucus, (4179) Toutatis, and 1994AW1 (Lupishko and Martino, 1998). We estimated the damping time scale of Nereus to be $700 \mathrm{Myr}$, with the equation by Burns and Safronov (1973) and the value of $\mu Q$ $\left(5 \times 10^{11} \mathrm{~Pa}\right)$ by Harris (1994), where $\mu$ is the rigidity and $Q$ is the quality factor of the asteroids. This time scale is comparable or longer than the average lifetime of NEOs (Bottke et al., 1994) or collisional lifetime of Nereus (Michel et al., 1996).

If the origin of Nereus is a small main-belt asteroid, the wobbling motion triggered by impacts in the main-belt must still remain today. If Nereus is an extinct comet nucleus, on the other hand, the excitation of wobbling motion by gas jetting may also be expected. However, it is possible that the value of $\mu Q$ for an extinct comet nucleus is much smaller than $5 \times 10^{11} \mathrm{~Pa}$. Therefore, if Nereus is an extinct comet nucleus, it can be explained that Nereus seems have no wobbling motion.

Since the duration of our observations was not sufficient enough to detect any wobbling motion, Nereus' wobbling motion was not confirmed. Further ground-based observation and/or exploration by spacecraft to elucidate the wobbling motion of Nereus will give some implications on the internal structure and its origin.

\section{Conclusion}

The photometric observation of Nereus has found out that the asteroid has a long rotational period, $15.1 \pm 1.2$ hours, and large amplitude of light variation. Its slow rotation is preferable for the spacecraft mission, but its highly elongated shape may have some problems on the mission scenario planning. The comparison with the previous data on SMBAs and comet nuclei implies that Nereus is more like an extinct comet than an asteroid thrown out from the main-belt. Its evaluated $\left(\frac{a}{b}\right)$ ratio is more elongated than the average ratio of laboratory impact experiments fragments. Spectral data of Nereus cannot still provide strong evidence about its origin. However, the featureless and flat spectrum observed by Tholen and Senay (1993), which suggests that the taxonomic type of
Nereus is neither S nor Q-type, is not inconsistent with the possible cometary origin of Nereus. Although there are some possibilities that rocky asteroids can be associated with meteor streams, meteors with orbits similar to that of Nereus are also a preferable evidence for the comet origin of Nereus. In any case, the present results indicate that Nereus is not only one of the most accessible asteroids but also a scientifically interesting object.

In January 2002, the Nereus will approach the Earth at the distance of 0.029 AU. Many ground-based observations including radar observations are expected to be performed at the opportunity.

Acknowledgments. It is a great pleasure to thank the staff of the European Southern Observatory (ESO) and the ESO/MPI 2.2-m telescope team on La Silla for their capable and gracious assistance. Special thanks go to M. Turatto and L. Ramirez of ESO La Silla. We also thank A. Fujiwara and H. Yano of ISAS, J. Watanabe and T. Fuse of NAOJ, R. P. Binzel of MIT, and D. J. Tholen of U. of Hawaii for suggestions to our observation and fruitful discussions on obtained results. Numerical computations were carried out on workstations at the Astronomical Data Analysis Center of the National Astronomical Observatory, Japan. Two anonymous referees for critical comments have improved the final version of this manuscript significantly.

\section{References}

Abe, M., H. Yamakawa, J. Kawaguchi, A. Fujiwara, and S. Yoshida, A brief survey of small body explorations in the early 2000, in Proc. 20th Inter. Symp. Space Tech. Sci., 96-i-12, 1996.

Babadzhanov, P. B., Asteroids and their meteor showers, in Seventy-Five Years of Hirayama Asteroid Families, edited by Y. Kozai, R. P. Binzel, and T. Hirayama, 168-185, Astron. Soc. Pacific, San Francisco, 1994.

Batrakov, Y. V. (Ed.), Ephemerides of Minor Planets for 1997, 110, 1996.

Binzel, R. P. and L. M. Sauter, Trojan, Hilda, and Cybele asteroids: New lightcurve observations and analysis, Icarus, 95, 222-238, 1992.

Binzel, R. P., S. Xu, S. J. Bus, and E. Bowell, Origins for the near-Earth Asteroids, Science, 257, 779-782, 1992a.

Binzel, R. P., S. Xu, S. J. Bus, and E. Bowell, Small main-belt asteroid lightcurve survey, Icarus, 99, 225-237, 1992 b.

Bottke, W. F., M. C. Nolan, R. Greenberg, and R. A. Kolvoord, Collisional lifetimes and impact statistics of near-Earth asteroids, in Hazards due to Comets \& Asteroids, edited by T. Gehrels, 337-357, Univ. Arizona Press, Tucson, 1994.

Bowell, E., B. Hapke, D. Domingue, K. Lumme, J. Peltoniemi, and A. W. Harris, Application of photometric models to asteroids, in Asteroid II, edited by R. P. Binzel, T. Gehrels, and M. S. Matthews, 524-556, Univ. Arizona Press, Tucson, 1989.

Burns, J. A. and V. S. Safronov, Asteroid nutation angles, Mon. Not. R. Astron. Soc., 165, 403-411, 1973.

Capaccioni, F., P. Cerroni, M. Coradini, P. Farinella, E. Flamini, G. Martelli, P. Paolicchi, P. N. Smith, and V. Zappalà, Shapes of asteroids compared with fragments from hypervelocity impact experiments, Nature, 308, 832-834, 1984.

Davis, D. R., A. L. Friedlander, and T. D. Jones, Role of Near-Earth Asteroids in the Space Exploration Initiative, in Resources of Near-Earth Space, edited by J. S. Lewis, M. S. Matthews, and M. L. Guerrieri, 619655, Univ. Arizona Press, Tucson, 1993.

Farquhar, R. W., D. W. Dunham, and J. V. McAdams, NEAR mission overview and trajectory design, J. Astronautical Sci., 43, 353-371, 1995.

Fujiwara, A., G. Kamimoto, and A. Tsukamoto, Expected shape distribution of asteroids obtained from laboratory impact experiments, Nature, 272, 602-603, 1978.

Fujiwara, A., J. Kawaguchi, T. Mukai, and K. Uesugi, Sample return mission to NEA: MUSES-C, Adv. Space Res., 25, 231-238, 2000.

Harris, A. W., Tumbling asteroids, Icarus, 107, 209-211, 1994.

Helin, E. F., N. D. Hulkower, and D. F. Bender, The discovery of 1982 DB, the most accessible asteroid known, Icarus, 57, 42-47, 1984.

Ishibashi, Y., M. Abe, and Y. Takagi, Photometric observation of (4660) Nereus, Adv. Space Res., 25, 277-280, 2000.

Jewitt, D., Cometary photometry, in Comets in the Post-Halley Era, edited 
by R. L. Newburn, Jr. et al., 19-65, Kluwer, Dordrecht/Boston, 1991. Kawaguchi, J., A. Fujiwara, and S. Sawai, Sample and return mission from asteroid Nereus via solar electric propulsion, Acta Astronautica, 38, 87$101,1996$.

Lafler, J. and T. D. Kinman, An RR Lyrae star survey with the Lick 20inch astrograph II. The calculation of RR Lyrae periods by electronic computer, Astrophys. J. Suppl., 11, 216-222, 1964.

Landolt, A. U., UBVRI photometric standard stars in the magnitude range $11.5<V<16.0$ around the celestial equator, Astron. J., 104, 340-376, 1992.

Lauer, M., Rosetta Asteroid Rendezvous and Flyby Opportunities, MAS Working Paper 388, European Space Operation Center, 1992.

Lomb, N. R., Least-squares frequency analysis of unequally spaced data, Astrophys. Space Sci., 39, 447-462, 1976.

Lumme, K. and E. Bowell, Radiative transfer in the surfaces of atmosphereless bodies. I. Theory, Astron. J., 86, 1694-1704, 1981a.

Lumme, K. and E. Bowell, Radiative transfer in the surfaces of atmosphereless bodies. II. Interpretation of phase curves, Astron. J., 86, 1705-1721, $1981 b$.

Lupishko, F. F. and M. Di. Martino, Physical properties of near-Earth asteroids, Planet. Space Sci., 46, 47-74, 1998.

Luu, J. X. and D. C. Jewitt, Near-aphelion CCD photometry of comet P/Schwassmann-Wachmann 2, Astron. J., 104, 2243-2249, 1992.

Michel, P., Ch. Froeschlé, and P. Farinella, Dynamical evolution of two near-earth asteroids to be explored by spacecraft: (433) Eros and (4660) Nereus, Astron. Astrophys., 313, 993-1007, 1996.

Murchie, S., J. Veverka, P. Thomas, J. Bell, M. Bell, B. Clark, A. Harch, P. Martin, D. Yeomans, M. Robinson, N. Izenberg, C. Chapman, W. Merline, L. McFadden, D. Wellnitz, and M. Malin, Imaging results from NEAR's flyby of 433 Eros, Abstract of Asteroids, Comets, and Meteors 1999, 13, 1999.

Ohtsuka, K., C. Shimoda, Y. Shiba, S. Okumura, M. Tomita, M. Abe, and J. Watanabe, A fireball related with asteroid (4660) Nereus?, in Proc. 17th. Solar System Sci. Symp., 32-35, Inst. Space Astronaut. Sci., Sagamihara, 1995 (in Japanese).
Olsson-Steel, D., Identification of meteoroid streams from Apollo asteroids in the Adelaide radar orbit surveys, Icarus, 75, 64-96, 1988.

Ostro, S. J., K. D. Rosema, and R. F. Jurgens, The shape of Eros, Icarus, 84, 334-351, 1990.

Pospieszalska-Surdej, A. and J. Surdej, Determination of the pole orientation of an asteroid. The amplitude-aspect relation revisited, Astron Astrophys., 149, 186-194, 1985.

Press, W. H., S. A. Teukolsky, W. T. Vetterling, and B. P. Flannery, Numerical Recipes in FORTRAN: The Art of Scientific Computing, Second Edition, 569-577, Cambridge Univ. Press, New York, 1992.

Tedesco, E. F. and J. Gradie, Discovery of M class objects among the nearEarth asteroid population, Astron. J., 93, 738-745, 1987.

Tholen, D. J. and M. C. Senay, Colorimetry of asteroids near and far; New results for Apollo asteroid (4660) Nereus and the trans-Saturnian object 1993 HA2, Bull. Am. Astron. Soc., 25, 1126, 1993.

Tholen, D. J. and R. J. Whiteley, (4660) Nereus among the Earth approaching asteroids, 32nd Scientific Assembly of COSPAR abstracts, 59, 1998.

Tody, D., The IRAF Data Reduction and Analysis System, in Instrumentation in Astronomy VI, edited by D. L. Crawford, Proc. SPIE, 627, 733$752,1986$.

Wetherill, G. W., Where do the Apollo objects come from?, Icarus, 76, 1-18, 1988.

Yeomans, D. K., Asteroid 433 Eros: The target body of the NEAR mission, J. Astronautical Sci., 43, 417-426, 1995.

Yoshikawa, M., Ch. Froeschlé, and P. Michel, Orbital evolution of two near-earth asteroids: Nereus and 1989ML, Adv. Space Res., 25, 273 276, 2000.

Zappalà, V., A. Cellino, A. M. Barucci, M. Fulchignoni, and D. F. Lupishko, An analysis of the amplitude-phase relationship among asteroids, Astron. Astrophys., 231, 548-560, 1990.

Y. Ishibashi (e-mail: isibasi@planeta.sci.isas.ac.jp), M. Abe (e-mail: abe@planeta.sci.isas.ac.jp), Y. Takagi (e-mail: takagi@toho-jc.ac.jp) 